\title{
Lumen
}

Selected Proceedings from the Canadian Society for Eighteenth-Century Studies

\section{Mettre en mouvement : conjecturalité et dialogisme chez Diderot}

\section{Mitia Rioux-Beaulne}

Volume 23, 2004

URI : https://id.erudit.org/iderudit/1012199ar

DOI : https://doi.org/10.7202/1012199ar

Aller au sommaire du numéro

Éditeur(s)

Canadian Society for Eighteenth-Century Studies / Société canadienne d'étude du dix-huitième siècle

ISSN

1209-3696 (imprimé)

1927-8284 (numérique)

Découvrir la revue

Citer cet article

Rioux-Beaulne, M. (2004). Mettre en mouvement : conjecturalité et dialogisme chez Diderot. Lumen, 23, 275-294. https://doi.org/10.7202/1012199ar

Copyright @ Canadian Society for Eighteenth-Century Studies / Sociéte canadienne d'étude du dix-huitième siècle, 2004
Ce document est protégé par la loi sur le droit d'auteur. L'utilisation des services d'Érudit (y compris la reproduction) est assujettie à sa politique d'utilisation que vous pouvez consulter en ligne.

https://apropos.erudit.org/fr/usagers/politique-dutilisation/ 


\section{Mettre en mouvement : conjecturalité et dialogisme chez Diderot}

\section{Introduction}

Le fait que les problèmes philosophiques les plus complexes soient, chez Diderot, la plupart du temps traités dans des écrits à haute teneur littéraire rend - cela a souvent été remarqué - malaisée la tâche de définir clairement sa position à l'égard de ces problèmes. L'écriture, même philosophique, a chez lui un tour déstabilisant : tout se passe comme si, volontairement, il cherchait à désorienter son lecteur. Coprésence de thèses contradictoires, dialogisme radical souvent aporétique, écriture fragmentaire ou allusive, interrogative ou ironique forment un style qui semble parfois confiner le lecteur à l'obscurité. La discursivité diderotienne laisse filtrer une conception de la pratique philosophique ${ }^{1}$ dont on n'a pas encore épuisé le sens.

Cette pratique est avant tout une pratique expérimentale, largement inspirée, certes, de la "philosophie expérimentale», du newtonianisme qui fait rage autour de lui, mais fortement empreinte d'une dimension éthique qui doit autant à son côtoiement de la philosophie shaftesburienne qu'aux Essais de Montaigne, dimension éthique qui valorise grandement la conversation et le dialogue dans l'activité philosophique. Cette pratique philosophique absorbe de même, voire radicalise, la procédure du doute méthodique inaugurée par la philosophie cartésienne, y voyant un moyen nécessaire pour échapper à tout dogmatisme. Les considérations sur le scepticisme dans les Pensées philosophiques (1746) montraient un Diderot assumant pleinement le caractère

1 Sur ce thème, voir, notamment, Georges Benrekassa, "La Pratique philosophique de Diderot dans l'article «Encyclopédie» de l'Encyclopédie», dans Stanford French Review, automne 1984, p. 189-212. Élisabeth de Fontenay, dans son Diderot : ou Le Matérialisme enchanté, Paris, Grasset, 1981, y fait également souvent référence. 
heuristique du doute. Seulement la réflexion sur la science de cette période n'avait pas encore recueilli les fruits du travail encyclopédique, et les quelques points de méthode qui sont annoncés sont, somme toute, formulés de manière assez laconique. Sept ans plus tard, dans ses Pensées sur l'interprétation de la nature, Diderot développera plus avant une épistémologie et une méthodologie dignes de ce nom. L'approche que Diderot y définit tente de concilier les méthodes qu'il appelle lui-même «expérimentale» et "rationnelle», et c'est alors qu'il mettra à profit une conception originale de la pratique philosophique qu'il a déjà mise en œuvre, dans la Lettre sur les aveugles (1749), par exemple, mais qu'il n'a pas encore réellement thématisée. Un concept trouve alors une place centrale dans cette approche, et Diderot l'emploiera jusque dans ses derniers écrits : la conjecture. La place de ce terme dans les Pensées sur l'interprétation de la nature est particulièrement intéressante, puisqu'il survient seulement dans leur seconde édition (1754), se substituant à celui de rêverie. Pour qui connaît la Lettre sur les aveugles et le Rêve de $d$ 'Alembert, cette substitution est éloquente : elle confirme que Diderot accorde à l'imagination une place importante et ce, jusque dans le travail de la science. Elle confirme, en quelque sorte, que l'écriture de ces textes est solidaire d'une méthode, qu'il y a une théorie de l'écriture philosophique chez Diderot.

On ne saurait prétendre, ici, faire le tour de la question : tout au plus, esquisser une analyse de la théorie de la conjecture développée dans les Pensées sur l'interprétation de la nature comme un cas de figure conférant un éclairage particulier sur les stratégies discursives déployées dans toute l'œuvre de Diderot. Dans un premier temps, il s'agira d'établir que la fonction de la conjecture est, à proprement parler, de mettre en mouvement la pensée du lecteur. Cette fonction s'exerce contre une attitude que Diderot exècre, qui est celle du dogmatisme, où se profile une vanité : celle de se croire l'unique détenteur d'une vérité définitive. Ainsi, on verra, dans un deuxième temps, que l'opération dynamisante de la conjecture repose sur une communauté de structure entre les sphères épistémologique et esthétique. Un des traits essentiels de la pensée de Diderot est cette idée qu'il y a une génialité à l'œuvre dans toutes les activités humaines, et que cette génialité est ce qui ouvre sans cesse des virtualités nouvelles. Enfin, il faudra faire le lien entre cette approche conjecturale et l'une des stratégies les plus accomplies chez Diderot, à savoir le dialogisme, si déroutant parfois - Le Neveu de Rameau est à cet égard l'exemple le plus probant. Il est peu d'endroits dans l'œuvre de Diderot où l'on trouve une théorisation explicite du dialogisme. Cependant, on trouve dans l'Apologie de l'abbé Galiani (1770), au-delà de la polémique économico-politique qui est son prétexte, une défense claire 
des vertus philosophiques du dialogue qui font directement écho à la pratique conjecturale.

\section{Fonctions épistémiques de la conjecture}

Les Pensées sur l'interprétation de la nature, en un sens, reprennent un problème abordé quatre ans plus tôt par Diderot dans la Lettre sur les aveugles. Ce problème, c'est celui d'une caractérisation du fait de la connaissance. Mais là où la Lettre cherchait une réponse du côté de la «génération» de nos idées, en en posant d'emblée la dépendance face à notre organisation physique, les Pensées offrent un déplacement de perspective. La Lettre, au fond, adoptait une approche assez fidèle à l'esprit de la «métaphysique» condillacienne, proposée dans l'Essai sur l'origine des connaissances humaines (1746) : il s'agissait alors de saisir les «opérations de l'esprit» pour en comprendre le fonctionnement et, autant que faire se peut, les mieux conduire. Mais le Diderot des Pensées, fort d'une expérience encyclopédique qui a exigé de lui des recherches moins spéculatives et l'a rapproché du travail des scientifiques, développe une approche beaucoup plus méthodologico-épistémologique. Il faut maintenant trouver les conditions de possibilité d'un discours scientifique cohérent et une méthode qui soit garante tant de sa validité que de sa fécondité.

Pour ce qui relève de la méthode, Diderot présente trois trajectoires possibles dans l'élaboration d'un savoir constitué, trois trajectoires possibles, c'est-à-dire deux manières habituelles de faire de la "philosophie» - toutes deux lacunaires - et une troisième - plutôt «éclectique» fondée sur une sorte de synthèse des deux premières, et qu'il entend ici promouvoir. La présentation des deux premières est offerte dès la première pensée, qui donne ainsi le ton de l'ouvrage : «Les uns [parmi «nos philosophes»] ont, ce me semble, beaucoup d'instruments et peu d'idées ; les autres ont beaucoup d'idées et n'ont point d'instruments ${ }^{2} . »$ Diderot les nommera explicitement philosophies «expérimentale» et "rationnelle ${ }^{3} »$. En un sens, son estime paraît plutôt pencher du côté de la philosophie expérimentale - celle-là même qui «a les yeux bandés» et

2 Denis Diderot, CEuvres, Laurent Versini (édit.), Paris, Robert Laffont, coll. «Bouquins», 1994-1997, 5 t. (Tous les renvois à l'œuvre de Diderot, à moins d'indication contraire, réfèrent à cette édition et seront notés de la manière suivante : Versini, [no. de tome], [no. de paragraphe, s'il y a lieu], [no. de page].) Ici, Versini, t. 1, p. 560.

3 Versini, t. 1, par. 23, p. 568. 
«marche toujours en tâtonnant» -, parce que son activité, comparable à celle des sens et à celle de l' «instinct», reste indéfectiblement attachée au réel, à l'extériorité. En l'occurrence, la philosophie expérimentale collectionne indéfiniment les phénomènes et correspond à cette activité originaire qui est celle du «manouvrier ${ }^{4} »$. Toutefois, s'il est vrai que cette "méthode» a pour avantage de s'en tenir aux faits, et s'il est vrai qu'à force d'expériences répétées, même les «manouvriers d'opérations les plus grossiers» peuvent développer un "pressentiment qui a le caractère de l'inspiration ${ }^{5}$ " - véritable démon familier socratique -, il n'en demeure pas moins que Diderot semble aussi penser qu'une telle philosophie rencontrera inévitablement un écueil, qui est celui de l'impossibilité et de l'inutilité d'une énumération exhaustive des phénomènes. Ainsi écrit-il :

La philosophie expérimentale travaillerait pendant les siècles des siècles, que les matériaux qu'elle entasserait, devenus à la fin par leur nombre au-dessus de toute combinaison, seraient encore bien loin d'une énumération exacte. Combien ne faudrait-il pas de volumes pour renfermer les termes seuls par lesquels nous désignerions les collections distinctes de phénomènes, si les phénomènes étaient connus? Quand la langue philosophique serait-elle complète? Quand elle serait complète, qui d'entre les hommes pourrait la savoir ${ }^{6}$ ?

À tout prendre, le défaut de la philosophie expérimentale est de manquer de réflexivité, entendue comme un travail de liaison des phénomènes entre eux.

Dans les considérations sur la philosophie rationnelle qu'on trouve dans les Pensées sur l'interprétation de la nature, Diderot adopte également une position ambivalente. Tantôt il la condamne, la considérant moins utile que la philosophie expérimentale qui amasse des faits, tantôt il la montre comme appartenant en propre au "génie" ${ }^{7}$. C'est qu'en effet,

4 L'article «Antédiluvienne (Philosophie)» affirme, faisant jouer l'Essai sur le mérite et la vertu de Shaftesbury contre Brucker que Diderot suit pourtant si souvent, que la philosophie, en tant que telle, est loin d'être une activité originaire de l'être humain, et que son développement dépend de ce que les besoins du corps aient été satisfaits. D'ailleurs, on notera que la plupart des articles sur l'«Histoire de la philosophie» montrent que, chez tous les peuples ayant développé quelque philosophie, tout a commencé par la rencontre de quelque phénomène sur une base expérimentale (voir, notamment, les articles «Chaldéens» et «Éthiopiens».).

5 Versini, t. 1, par. 30, p. 570.

6 Versini, t. 1, par. 6, p. 562.

7 Versini, t. 1, par. 21, 23, p. 568. 
travaillant avec les matériaux de la philosophie expérimentale, elle produit des édifices fragiles qui finissent par s'écrouler avec le temps qui apporte de nouveaux faits. Mais, d'autre part, ces constructions demandent une imagination forte, une grande éloquence, un art de présenter ses idées en images fortes et sublimes ${ }^{8}$. L'erreur des philosophes rationnels - nous y reviendrons un peu plus loin - consiste en ceci qu'ils s'attachent trop peu à ce que fournit l'expérience. Ils omettent de mettre leurs idées à l'épreuve du réel, développant ces «billevesées de la métaphysique» que condamnaient déjà les Pensées philosophiques ${ }^{9}$. L'ambivalence de Diderot définit au fond l'interdépendance et la complémentarité des deux méthodes. Le problème vient de ce que la nature ne laisse échapper que des parties d'elle-même, les phénomènes n'apparaissant que détachés les uns des autres ${ }^{10}$. Seule l'imagination peut s'efforcer de recomposer les liens qui les unissent. Ainsi se constitue donc la chaîne épistémique sans cesse réitérée tout au long des Pensées sur l'interprétation de la nature : partant des faits observés, on peut établir conjecturalement leur liaison, que seule l'expérience peut vérifier. Véritable aller-retour entre l'extériorité et l'intériorité, entre l'«instinct» et la «raison» : «Tout se réduit à revenir des sens à la réflexion, et de la réflexion aux sens : rentrer en soi et en sortir sans cesse ${ }^{11}$.»

$C^{\prime}$ est dans ce contexte, donc, que se laisse penser le concept de ce qu'entend Diderot par «conjecture». La conjecture correspond au travail réflexif qui s'opère sur les données fournies par la philosophie expérimentale. Ce travail est de l'ordre de la combinatoire : «L'observation recueille les faits, la réflexion les combine, l'expérience vérifie le résultat de la combinaison ${ }^{12}$.» Et son rôle, dès lors, est avant tout heuristique : en combinant les faits, la conjecture systématise les résultats d'une démarche empirique. Or, cette systématisation, Diderot la comprend non pas, en tant que telle, comme une production de connaissance, mais comme l'ouverture d'une connaissance possible. Pour passer au rang de connais-

8 Versini, t. 1, par. 21, 23, p. 568.

9 Versini, t. 1, par. 17, p. 22.

10 Voir Versini, t. 1, par. 11, 12, p. 564-565, où Diderot affirme la continuité nécessaire de la chaîne des phénomènes et la manière fragmentaire dont cette chaîne nous apparaît.

11 Versini, t. 1, par. 9, p. 564. Le par. 10 dit : «Mais par malheur il est plus facile et plus court de se consulter soi que la nature. Aussi la raison est-elle portée à demeurer en elle-même, et l'instinct à se répandre au-dehors.» 
sance avérée, la conjecture doit être soumise à l'épreuve du réel : «Tant que les choses ne sont que dans notre entendement, ce sont nos opinions ; ce sont des notions qui peuvent être vraies ou fausses, accordées ou contredites. Elles ne prennent de la consistance qu'en se liant aux êtres extérieurs $^{13} . »$ Sans quoi, la conjecture reste une pure abstraction, restant, comme les mathématiques, «un monde intellectuel», coupé de la «terre ${ }^{14}{ }$. La fonction heuristique de la conjecture vient donc de ce qu'elle permet de transcender l'immédiateté de l'expérience présente en la mettant en rapport avec des expériences passées ou possibles. Elle rend possible la constitution d'un horizon de compréhension des faits isolés en les reconduisant à leur enchaînement conçu. Aussi la conjecture doit-elle être comprise comme un succédané : elle se substitue aux liaisons dont on ne peut faire l'expérience. Sa construction n'a rien d'arbitraire, puisqu'elle doit toujours être le résultat d'une combinaison de matériaux expérimentés, seulement sa valeur d'objectivité n'est pas garantie - elle n'a pas le caractère de nécessité qui est celui des liaisons effectives entre les phénomènes - puisque, justement, elle est une simple reconstruction imaginaire de ce qui n'est pas donné dans l'expérience. Diderot mobilise ici le critère de "consistance» pour en relativiser la valeur d'objectivité : cette consistance désigne le fait que les expériences confirment ce que l'on devrait être en droit d'attendre des phénomènes suivant une conjecture donnée.

À proprement parler, une telle approche méthodologique semble suivre la ligne tracée par la «méthode expérimentale» classique suivie par la plupart des philosophes adeptes de Newton. Pourtant, Diderot imprime quelque chose dans sa théorie de la conjecture qui outrepasse les recommandations de "sobriété philosophique» du newtonianisme, tant valorisée par Voltaire, pour ne nommer que lui ${ }^{15}$. Là où Voltaire relègue au rang de fable ou de roman les philosophies fondées sur une imagination féconde et aveugle - comme celle de Descartes - , Diderot

13 Versini, I, par. 7, p. 563, je souligne. Voir aussi l'article «Épreuve, essai, expérience»: «Termes relatifs à la manière dont nous acquérons la connaissance des objets. Nous nous assurons par l'épreuve, si la chose a la qualité que nous lui croyons.»

14 Les par. 2 et 3 offrent une critique en règle de la valeur de vérité des mathématiques qui fait écho à certains passages de la Lettre sur les aveugles (voir Versini, t. 1, p. 151-152), réservant la réduction à des unités numériques aux intelligences pures. L'article «Abstraits (en logique)», écrit par Diderot, dit : «Les termes abstraits, ce sont ceux qui ne marquent aucun objet qui existe hors de notre imagination.»

15 Voir Éliane Martin-Haag, Voltaire, Paris, Vrin, coll. «Bibliothèque des philosophes», chap. 1 et 2, 2002, p. 17 à 38 . 
fait intervenir la notion de conjecture qui leur redonne droit de cité dans une histoire de la science en tant qu'expériences de pensée ayant eu leur utilité. En effet, Diderot ne s'inquiète pas des extravagances des philosophes. Au contraire, même, il la commande : «Je dis extravagances : car quel autre nom donner à cet enchaînement de conjectures fondées sur des oppositions ou des ressemblances si éloignées, si imperceptibles, que les rêves d'un malade ne paraissent ni plus bizarres, ni plus décousus ${ }^{16}$ ?» La seule restriction est posée comme un après-coup et tient à ce critère de consistance évoqué plus haut : il s'agit de ne pas tenir plus aux conjectures qu'aux faits, de laisser à ceux-ci le dernier mot. Sur le plan d'une heuristique, en conséquence, il s'agit de ne rien présupposer sur la nature - ce qui veut aussi dire ne pas lui imposer de restriction — et de laisser s'éprouver toutes les possibilités.

Cependant, il reste (au moins) une ambiguïté dans cette théorie de la conjecture, ambiguïté qui n'est pas thématisée comme telle par Diderot, mais que l'on décèle en observant l'usage qu'il en fait dans les Pensées sur l'interprétation de la nature. C'est qu'en fait il y a deux types de conjecture. Le premier répond indubitablement à cette exigence scientifique et heuristique de systématisation des données obtenues par l'observation en vue d'imaginer des expériences ultérieures de confirmation ou de falsification de la conjecture. Une série d' «exemples» de ce type de conjecture forme le groupe des pensées 32 à 38, portant sur des problèmes de biologie, de physique, de chimie, etc. Dans ces exemples, Diderot suit à la lettre la méthodologie qu'il préconise : partant du savoir disponible, il imagine des expériences diverses qui devraient permettre de décider en faveur ou en défaveur d'une théorie hypothétique échafaudée sur la base de ce savoir. On comprendra que ce type de conjecture scientifique a pour fonction principale de dynamiser le travail des «naturalistes». Elle correspond à peu près à ce que nous nommerions aujourd'hui une théorie scientifique. Diderot assume même que l'épreuve de l'expérience, quand bien même elle infirmerait la théorie, ne doit pas la faire rejeter trop rapidement : «Quand on a formé dans sa tête un de ces systèmes qui demandent à être vérifiés par l'expérience, il ne faut ni s'y attacher opiniâtrement, ni l'abandonner avec légèreté. On pense quelquefois de ses conjectures qu'elles sont fausses, quand on n'a pas pris les mesures convenables pour les trouver vraies ${ }^{17} .{ }^{\prime \prime}$ 
Mais il est un second type de conjecture ou de rêverie dont use Diderot, et qui semble avoir une portée autrement plus profonde : c'est la conjecture ontologique ou métaphysique, celle qui unifie le savoir en une image de la totalité de la nature. Il apparaît improbable - et ce, même à Diderot - qu'un jour une telle conjecture vienne à être $a b$ solument confirmée. Les pensées 11 et 12 , à cet égard, sont des plus éclairantes. Dans la pensée 11, Diderot affirme que «l'indépendance absolue d'un seul fait est incompatible avec l'idée de tout ; et sans l'idée de tout, plus de philosophie ${ }^{18} . »$ Cette idée sert à renforcer la thèse selon laquelle la nature «n'a peut-être produit qu'un seul acte ${ }^{19}$.» La «chaîne des êtres» demeure, en ce sens, une conjecture. De même, dans la pensée 12, Diderot affirme que la conjecture suivant laquelle il y a un prototype de tous les êtres, qu'elle soit vraie, comme le veut Baumann ${ }^{20}$, ou fausse, suivant Buffon, reste «essentielle au progrès de la physique expérimentale, à celui de la philosophie rationnelle, à la découverte et à l'explication des phénomènes qui dépendent de l'organisation ${ }^{21}{ }^{\prime}$. Au-delà de son caractère heuristique, la conjecture prend ici une fonction constitutive pour la science, indépendamment de sa valeur de vérité. Elle détermine le point de vue à partir duquel la nature doit être envisagée pour être susceptible de compréhension. Elle forme, à proprement parler, un horizon d'explication des phénomènes. À ce titre, pourtant, elle vise au-delà de ce qui relève d'une simple conjecture de type scientifique, dans la mesure où elle ne renvoie pas directement à des expériences de confirmation et de falsification.

La conjecture métaphysique, en dernière analyse, développe ce qui relève de la construction d'un point de vue proprement philosophique sur le monde, dont il offre une vue unifiée. Ce trait - celui d'identifier vue unifiée du monde et discursivité philosophique - est récurrent dans l'œuvre de Diderot depuis sa traduction de l'Essai sur le mérite et la vertu de Shaftesbury (1745), où il affirme déjà dans une note que le principe selon lequel «dans l'univers, tout est uni» est le premier pas de la philosophie, supposé par tous les «auteurs de système», ajoutant que "plus on voit loin dans la nature et plus on y voit $\mathrm{d}^{\prime}$ union $^{22}$.» Dès lors, il faut prendre au sérieux le fait qu'il s'agit là de

18 Versini, t. 1, p. 564.

19 Versini, t. 1, p. 564, je souligne.

20 Pseudonyme de Maupertuis.

21 Versini, t. 1, p. 565.

22 Voir l'édition de l'Essai au tome 1 des CEuvres complètes de Diderot, Dieckmann, 
suppositions ou de conjectures qui n'ont pas la "consistance» des vérités scientifiques. Il faut prendre au sérieux le fait que le matérialisme de Diderot n'est pas du même ordre que celui d'un La Mettrie, par exemple, puisqu'il reste, avant tout, un modèle explicatif, découlant de ce que Jean-Claude Bourdin nomme une "stratégie épistémologiquement faible ${ }^{23}$ » principalement fondée sur la démonstration du caractère non nécessaire d'un recours à une matière spirituelle pour l'explication des phénomènes.

Mais malgré la différence de fonction qui articule la distinction entre ces deux types de conjectures, il importe de ne pas trop les séparer. En un sens, elles relèvent toutes deux du même mécanisme de liaison des phénomènes. Il y a surtout, de l'une à l'autre, ce qu'il y a du particulier au général. La conjecture scientifique porte sur un ensemble de phénomènes particulier et cherche les principes qui lient ces phénomènes $-c^{\prime}$ est ce qui la rend apte à prévoir des résultats et à être éprouvée par des expériences. La conjecture métaphysique porte sur la totalité des phénomènes et ne pourrait être définitivement confirmée qu'une fois la totalité du savoir acquise - ce qui est, Diderot l'a admis, sans doute au-delà des bornes de l'entendement. Cette différence se creuse légèrement au moment de déterminer leurs objets respectifs. La pensée $56^{24}$ donne un aperçu de la manière dont Diderot entrevoit le problème. Il y est demandé au physicien d'abandonner le pourquoi au profit du comment. C'est que le comment «se tire des êtres». Autrement dit, le travail du scientifique ne demande qu'une relative fidélité à ce qui est empiriquement observé. Le pourquoi, pour sa part, "se tire de l'entendement», «tient à nos systèmes» et "dépend du progrès des connaissances». C'est-à-dire que le travail philosophique ou métaphysique est essentiellement un travail réflexif sur ce qui est fourni par le travail scientifique. Constitué a posteriori, un tel horizon de compréhension n'atteint pas à la consistance des vérités scientifiques : y croire signifierait «substituer la conjecture de l'homme à l'ouvrage de Dieu... attacher la plus importante des vérités au sort d'une hy-

Proust, Varloot (édit.), Paris, Hermann, 1975, p. 313 (à l'avenir : DPV, [no. de tome], [no. de page].). Dans une autre note (p. 359 de la même édition), Diderot parle du «regard du philosophe» : voyant la grandeur de l'univers, tout lui apparaît petit et sans importance - il se trouve alors «noyé» dans l'immensité des êtres.

23 Voir Jean-Claude Bourdin, «Les vicissitudes du moi dans Le Rêve de d'Alembert de Diderot", dans Jean-Noël Missa (édit.), Matière pensante. Études historiques sur les conceptions matérialistes en philosophie de l'esprit, Paris, Vrin, 1999, p. 66-67.

24 Versini, t. 1, p. 594. 
pothèse ${ }^{25} . »$ La vérité philosophique, entendue comme constitution d'un horizon d'interprétation des phénomènes, reste essentiellement dans le domaine du possible.

\section{Une analogie de structure : épistémologie et esthétique}

Le caractère conjectural en dernière instance de toute vue totalisante permet à Diderot de rester en accord avec l'arrière-fond sceptique à $l^{\prime} œ u v r e$ dans toute sa pensée ${ }^{26}$. Scepticisme que la fin de la Lettre sur les aveugles clamait déjà : "Hélas! Madame, quand on a mis les connaissances humaines dans la balance de Montaigne, on n'est pas éloigné de prendre sa devise. Car, que savons-nous ? ce que c'est que la matière ? nullement; ce que c'est que l'esprit et la pensée ? encore moins ; ce que $c^{\prime}$ est que le mouvement, l'espace et la durée ? point du tout ${ }^{27}$...» L'enjeu, au fond, consiste en ceci qu'il s'agit d'éviter les erreurs d'une philosophie rationnelle outrancière qui se croirait à même de faire l'économie des résultats de la méthode expérimentale - qui se croirait, au fond, capable d'atteindre l'essence des choses par la seule force de l'esprit.

Les critiques que Diderot porte, tout au long des Pensées sur l'interprétation de la nature, à l'endroit de la philosophie rationnelle montrent clairement que le problème de cette philosophie en est un de vanité. Il y a une limite au-delà de laquelle l'opiniâtreté recommandée par Diderot dans la mise à l'épreuve d'une théorie tourne au dogmatisme :

Quand on suit une mauvaise route, plus on marche vite, plus on s'égare; et le moyen de revenir sur ses pas, quand on a parcouru un espace immense ? L'épuisement des forces ne le permet pas ; la vanité s'y oppose sans qu'on s'en aperçoive ; l'entêtement des principes répand sur tout ce qui environne un prestige qui défigure les objets. On ne les voit plus comme ils sont, mais comme

25 Déjà, l'exergue des Pensées rappelait la nécessité de rester vigilant pour ne pas confondre deux ordres de réalité : «Une hypothèse n'est pas un fait.» Versini, t. 1, p. 559.

26 Cet «arrière-fond sceptique» est celui, au fond, de la plupart des philosophes des Lumières et porte principalement sur l'impossibilité, pour l'être humain, d'accéder à un savoir «transcendant». Voir Giorgio Tonelli, «The «Weakness» of Reason in the Age of Enlightenment», dans Richard Henry Popkin, Ezequiel de Olaso et Giorgio Tonelli (édit.), Scepticism in the Enlightenment, Dordrecht, Kluwer Academic, 1997, p. 35-50.

27 Versini, t. 1, p. 184. 
il conviendrait qu'ils fussent. Au lieu de réformer ses notions sur les êtres, il semble qu'on prenne à tâche de modeler les êtres sur ses notions ${ }^{28}$.

L'idée d'un tel glissement de l'opération réflexive à la croyance en la réalité de ce qui devrait être laissé au rang de conjecture correspond très exactement au glissement qui s'opère dans le développement de la superstition, tant décriée par Diderot. Un détour par l'article «Éthiopiens (Philosophie des)» de l'Encyclopédie l'illustre. Dans cet article, Diderot explique que les Éthiopiens ont inventé l'astronomie, assurant, par exemple, que la lumière de la lune est «empruntée», après avoir observé l'inconstance de la lune et s'en être étonnés. Ce premier mouvement correspond, on le voit, au travail scientifique, partant d'observation et s'élevant au rang de formulations générales. Alors survient le glissement vers le "préjugé», au point précis où ces formulations, de conjectures qu'elles étaient, passent au rang de «vérité». «Les révolutions, écrit Diderot, et même les irrégularités des autres corps célestes ne leur échappèrent pas ; ils formèrent des conjectures sur la nature de ces êtres ; ils en firent des causes physiques générales. Ils leurs attribuèrent différents effets, et ce fut ainsi que l'Astrologie naquit parmi eux de la connaissance astronomique.» — où «Astrologie» doit s'entendre comme une forme de superstition. Des physiciens qui se laissent aller à un tel glissement, les Pensées disent explicitement qu' $^{\prime}$ «ils se sont abandonnés à la superstition de leurs conjectures ${ }^{29} . »$

Ce qui est du plus grand intérêt, ici, c'est ce qui différencie la philosophie rationnelle, comprise comme pendant rationaliste de la superstition religieuse, de l'approche conjecturale, à savoir que dans la première, c'est surtout la vanité humaine qui se manifeste, la prétention à détenir une certitude sur les fins de la nature, vanité que prétend mettre en échec la procédure conjecturale par sa forme même. Le simple fait d'appeler «rêverie» ou "conjecture» tous les développements rationnels opérés à partir des faits, tous les «systèmes» de la philosophie rationnelle, toutes les liaisons imaginées entre les phénomènes les plus divers rappelle constamment au lecteur leur caractère d'extravagance. Et de la Lettre sur les aveugles au Rêve de d'Alembert, en passant par les Entretiens sur le Fils naturel et Le Neveu de Rameau, Diderot prend toujours soin, alors qu'il explicite ses idées sur l'unité de la nature, de placer son discours dans la bouche d'un individu en proie à un délire inspiré, d'un enthousiaste au 
tour d'esprit poétique ou frivole. Le caractère hypothétique du matérialisme de Diderot se trouve ainsi, performativement en quelque sorte, sans cesse réaffirmé. Et, sur cette base, la différence apparaît entre la conjecture et le dogmatisme, qui réside principalement dans la manière de se rapporter aux idées qui naissent en nous.

De la même façon se creuse la distinction entre deux formes d'enthousiasme, où l'on peut voir un écho de la Letter Concerning Enthusiasm de Shaftesbury ${ }^{30}$. Le dogmatisme philosophique pécherait par le même excès que l'enthousiasme religieux, à savoir qu'il prétendrait pouvoir établir un contact immédiat avec l'essence des choses. Il faut, à ce titre, se reporter au désormais célèbre article "Théosophes» de l'Encyclopédie. Diderot y reprend l'idée selon laquelle une faculté de pressentir anime variablement les êtres humains, suivant leur organisation et l'expérience qu'ils ont acquise, qui leur confère une certaine "prudence» les poussant à prévoir les résultats à escompter d'une situation donnée. «Ils rapprochent les analogies les plus éloignées; ils voient des liaisons presque nécessaires où les autres sont loin d'avoir des conjectures.» Si Diderot prend sur lui de défendre la valeur d'une telle "prudence», qui est, à n'en pas douter, celle du génie, cela ne va pas sans restrictions : «Je conjecture que ces hommes, d'un tempérament sombre et mélancolique, ne devaient cette pénétration extraordinaire et presque divine qu'on leur remarquait par intervalles, et qui les conduisait à des idées tantôt si folles, tantôt si sublimes, qu'à quelque dérangement périodique de la machine $^{31} . » C^{\prime}$ 'est que, ajoute Diderot, «le génie et la folie se touchent de bien près.» Qu'est-ce à dire ? Les théosophes, au fond, sont présentés de manière à montrer la pertinence philosophique d'une «imagination ardente», en tant qu'elle confère une génialité ; mais, en retour, Diderot persiste dans l'idée selon laquelle les théosophes doivent être rangés du côté de la superstition justement parce qu'ils ont trop confiance dans leurs vues : «ils se prétendirent éclairés par un principe intérieur, surnaturel et divin.»

La théorie diderotienne de la conjecture repose aussi sur une conception de l'inspiration et de la génialité comme faculté de lier des phénomènes éloignés. C'est pourquoi elle peut à bon compte récupérer le concept d'une "prudence» se développant jusqu'à l'extravagance. Mais ce dont elle ne saurait se passer, c'est de la nécessité d'une mise à

30 Voir John M. Robertson (édit.), Characteristics of Men, Manners, Opinions, Times, Indianapolis-New York, The Bobbs-Merrill Company, Inc., 1964, p. 5-39.

31 Je souligne. 
l'épreuve par le jeu d'une confrontation avec le monde extérieur. On doit ici noter qu'une telle confrontation, si elle a été jusqu'ici définie dans le cadre d'une expérimentation scientifique, s'étend aussi à une confrontation avec les idées d'autrui. Au reproche d'obscurité que Diderot assène à la théosophie correspond une exigence, manifestée dans les Pensées sur l'interprétation de la nature ${ }^{32}$, de clarté et de divulgation. Partout où il propose ses propres conjectures, Diderot demande en quelque sorte à son lecteur d'en éprouver la consistance par lui-même. En contrepartie, il s'efforce de faire de même avec les théories d'autrui ${ }^{33}$. En ce sens, la conjecture déploie déjà une conception essentiellement dialogique de la pratique philosophique, instaurant, du même coup, une communauté liant le philosophe et ceux à qui il s'adresse. Ainsi, aussi enthousiaste la génialité du philosophe soit-elle, elle ne confère à son discours aucune autorité a priori. Là est la pierre de touche d'une approche conjecturale, que l'exergue des Pensées résume ainsi : «Comme je me suis moins proposé de t'instruire que de t'exercer, il m'importe peu que tu adoptes mes idées ou que tu les rejettes, pourvu qu'elles emploient toute ton attention. Un plus habile t'apprendra à connaître les forces de la nature ; il me suffira de $t^{\prime}$ avoir fait essayer les tiennes.»

Essentiellernent, la conjecture, combinant des éléments disparates, doit «dynamiser» le lecteur auquel elle s'adresse en lui faisant voir des liaisons inouïes pour lui. La théorie des renvois, "partie de l'ordre encyclopédique la plus importante», dans l'article «Encyclopédie ${ }^{34}$ » montre ce qu'il en est de cette dynamisation : opérant des liaisons entre les entrées du dictionnaire, les renvois fonctionnent de la même façon que la conjecture. Or, Diderot assume clairement que l'effet produit par ces renvois est de "changer la façon commune de penser». La dynamique des renvois en est une à la fois de découverte, de démonstration de l'unité de la nature et d'ébranlement des idées reçues. C'est là un aspect de la multiplication des conjectures qui est crucial : elle empêche le savoir de se figer en interpellant le lecteur et en lui demandant $d^{\prime}$ '«essayer», sans cesse, ses forces. Le risque que court toujours l'enthousiasme, la génialité, mais aussi la philosophie rationnelle, est celui d'un repli sur soi qui signifierait une véritable interruption de la pensée. Si un système se

32 Versini, t. 1, par. 39-41, p. 580-582.

33 Les Pensées interrogent sans cesse les philosophies de contemporains, et principalement celle de Maupertuis, avec qui Diderot dialogue en de nombreux endroits.

34 Voir, dans l'édition Versini, t. 1, p. 402-403. 
construit, au fond, de la même manière qu'une conjecture, son défaut est de se croire définitif. Il introduit ainsi une rupture dans le commerce des idées et, sur le plan éthique, une rupture de la socialité. En ce sens, la vertu de la conjecture est justement de s'auto-affirmer comme une vue de l'esprit qui demande à être éprouvée par autrui.

Ces rapprochements entre conjecturalité et génialité appellent quelques remarques sur l'analogie que Diderot semble supposer entre les sphères épistémologique et esthétique. Si le génie philosophique se manifeste par la découverte de rapports entre les phénomènes, rien ne le sépare du génie artistique. Le Traité $d u$ beau (1751), édition séparée de l'article «Beau» de l'Encyclopédie, en effet, définissait le beau dans les termes mêmes qui seront ceux de la définition de la conjecture : la perception du beau est une perception de rapports ${ }^{35}$. Cette approche cognitive de la perception du beau est intimement liée à l'idée que l'unité de la nature y apparaît avec une sorte de fulgurance dont est tributaire l'enthousiasme propre au génie. En ce sens, il y a effectivement analogie de structure entre la perception du beau et la perception du vrai, pour peu qu'on réserve au terme de «vrai» un sens faible. C'est-à-dire que l'enthousiasme suscité par les rapports perçus reste un enthousiasme pour une «vue» de l'esprit qui n'a pas encore acquis la consistance d'une vérité scientifique. Dans sa lettre-préface à Grimm au Salon de 1767, élaborant sa théorie du «modèle idéal», Diderot fonde le critère de la beauté sur l'imitation d'un tel modèle ${ }^{36}$. Or, le propre du génie est justement de s'élever, suite à une inspiration ou par tâtonnements, jusqu'à percevoir ce modèle idéal, «ligne vraie qui n'exista nulle part que dans la tête des Agasias, des Raphaëls, des Poussins, des Pugets, des Pigalles, des Falconets ${ }^{37}$.» Aussi Diderot affirme-t-il : "Convenez donc que, quand vous faites beau, vous ne faites rien de ce qui est, rien même de ce qui puisse être ${ }^{38}$.»

La conjecture, en somme, peut se laisser comprendre comme une sorte de modèle épistémologique idéal. Elle constitue un modèle intellectuel des rapports effectifs qui lient les phénomènes. Suivant ainsi, peut-être mieux encore que nombre de philosophes de son temps, le requisit méthodologique du newtonianisme, Diderot refuse de conférer un statut

35 On trouvait déjà une définition de cet ordre dans les Mémoires sur différents sujets de mathématiques (1748: DPV, t. 2, p. 236-237.)

36 Versini, t. 4, p. 521-527.

37 Versini, t. 4, p. 525, je souligne.

38 Versini, t. 4, p. 524. 
de réalité à une vérité qui n'est jamais qu'une représentation du monde, représentation entièrement dépendante de l'organisation de l'être qui la construit. La loi de l'attraction demeure un «modèle» mathématique rendant compte de la constance des phénomènes, elle ne désigne pas une force réelle à l'œuvre dans la nature. Elle n'acquiert de consistance qu'à être toujours soumise à expérimentation et à révision ${ }^{39}$. Elle forme ainsi une sorte de conjecture ouvrant un horizon de compréhension. Mais cet horizon reste une proposition qu'il faut se garder d'élever au rang de dogme.

\section{L'art du dialogue}

Cette sorte d'auto-limitation du discours conjectural est justement ce qui caractérise la forme dialogique qui prend une place si importante dans l'œuvre de Diderot. Ce n'est certes pas le lieu, ici, pour interroger à fond la question du dialogisme chez Diderot, question qui a déjà fait l'objet de nombreux travaux et qui reste beaucoup trop complexe dans toutes ses implications pour être embrassée par un coup d'œil si rapide. Je voudrais plutôt ici esquisser quelques remarques qui permettent d'articuler la question du dialogisme à partir des considérations précédentes sur la théorie de la conjecture. Dans son Apologie de l'abbé Galiani ${ }^{40}$, Diderot thématise la question du dialogue comme il l'a peu fait ailleurs dans son œuvre. Or, on verra que l'apologie de l'abbé Galiani y prend l'allure, surtout, d'une apologie des vertus d'une parole qui ne tend pas à s'imposer comme détentrice de vérité.

Essentiellement, l'Apologie entend défendre, contre la réfutation de l'abbé Morellet, le Dialogue sur les blés de Galiani. Ce faisant, il défend une œuvre où il admet avoir trouvé des erreurs contre sa réfutation qu'il considère, en substance, juste. Si l'avantage revient tout de même à Galiani, c'est à cause des choix formels des deux auteurs : 1'un a privilégié le dialogue, l'autre a le ton monologique de la raison :

39 Diderot, d'ailleurs, ne suivait pas Newton sur toutes ses positions. S'il accepte l'attraction, il refuse l'hypothèse du vide que Newton croit nécessaire d'y adjoindre. Voir Aram Vartanian, "Diderot et Newton», dans Claude Blanckaert, Jean-Louis Fischer et Roselyne Rey (édit.), Nature, histoire, société. Essais en hommage à Jacques Roger, Paris, Klincksieck, 1995, p. 61-78.

40 Ce texte, portant sur des questions d'économie politique, n'a pas vraiment fait l'objet d'études dans l'axe proposé ici. 
[La Réfutation de Morellet] est l'ouvrage d'un homme de sens, et l'ouvrage de l'abbé Galiani est celui d'un homme de génie. Tout ce que celui-ci dit, bon ou mauvais, faux ou vrai, lui appartient. Il n'y a pas un mot qui soit à l'autre. Il n'y a que Galiani qui pût faire son livre ; tout le monde aurait fait la Réfutation de Morellet. L'abbé Galiani pense et nous fait penser ; l'autre se fait écouter parce qu'il a raison, mais il ne vous fait pas penser parce qu'il ne pense pas et qu'il vous fatigue et vous ennuie. [...] En ne disant que des choses communes, il a plus de morgue que Montesquieu. [...] L'abbé Morellet n'a pas un grain de l'enthousiasme de la gloire, de la véritét ${ }^{41}$.»

Le fond de la critique que Diderot adresse à Morellet tient à ceci : Galiani sait nous faire penser, et cela ne tient pas nécessairement à sa rigueur analytique, voire au fait que ce qu'il dit soit vrai, mais à sa manière - à son "génie». La vertu de ce génie est justement celle de l'effet $q u^{\prime} i l$ produit sur nous, à savoir celui de susciter une activité de l'esprit ${ }^{42}$. Et cette vertu lui est conférée par le caractère inouï de ce que présente Galiani, opposé aux idées "communes» de Morellet. Il y a là, au fond, quelque chose de l'ordre d'un critère qui permet de distinguer ce que Diderot juge être une attitude éminemment philosophique d'une attitude platement dogmatique, ce qu'explicite une série d'oppositions où réapparaît un motif rencontré plus tôt :

Si vous avez pris pour indifférence cet air d'impartialité qu'il a dû avoir dans les Dialogues, qui séduit, qui captive, qui entraine, tant pis pour vous. C'est que vous avez beaucoup de morgue et peu de goût ; $c^{\prime}$ est que vous êtes dogmatique et que l'abbé est enquêtant ; c'est que vous aimez la dispute et qu'il aime la causerie ; c'est que vous êtes toujours sur les bancs d'école et que l'abbé est toujours sur un canapé ${ }^{43}$.

Rarement Diderot a-t-il plus explicitement divulgué le fond de la stratégie dialogique, qu'il a si souvent lui-même mise en œuvre : affec-

41 Versini, t. 3, p. 124.

42 Il y a une tradition du «faire penser» dans le domaine de l'esthétique. On en voit déjà une formulation chez Du Bos, par exemple. Voir ses Réflexions critiques sur la poésie et sur la peinture, Paris, École nationale supérieure des Beaux-Arts, 1993, part. 1, sec. 6, p. 18 : «C'est ainsi qu'en ont usé le Poussin, Rubens et d'autres grands maîtres, qui ne se sont pas contentés de mettre dans leurs paysages un homme qui passe son chemin, ou bien une femme qui porte des fruits au marché. Ils y placent ordinairement des figures qui pensent afin de nous donner lieu de penser..." Je souligne.

43 Versini, t. 3, p. 125. 
tation d'impartialité dans le but de séduire, captiver et entraîner, mais non pas de persuader, puisqu'il s'agit de la méthode d'un homme enquêtant, aimant la causerie, opposée à celle d'un homme dogmatique fait pour la dispute. Le dialogisme prôné ici est de l'ordre du commerce des idées où chacun expose ses vues pour autrui. Il s'élève ainsi au rang d'exemple d'une méthode qui commande de ne pas accorder à nos "conjectures", aussi enthousiasmantes soient-elles, plus de réalité qu'elles n'en peuvent avoir, au rang d'une véritable éthique de la recherche philosophique qui confère au dialogue le statut, pour reprendre une expression de L. G. Crocker, de véritable «expérience morale ${ }^{44}$ ».

Par ailleurs, les accusations que Diderot laisse tomber sur Morellet, tout au long de l'Apologie, concernent le caractère abstrait, coupé de l'expérience, de ses idées ${ }^{45}$. Et la défense de l'abbé Galiani revient constamment sur le fait que les idées contenues dans son dialogue sont en parfaite unité avec les personnages en présence - et que rien n'indique qu'il faille les prendre pour les idées de Galiani lui-même. C'est là ce que Diderot appelle «l'art du dialogue» - auquel un dogmatique comme Morellet «n'entend rien ${ }^{46}$ ». Art qui donne à la pratique de la philosophie un sens de recherche. L'apologie de la manière de Galiani, au fond, correspond à la défense d'un principe dont Diderot se réclamait dès 1746, dans les Pensées philosophiques, à savoir qu'«on doit exiger de moi que je cherche la vérité, mais non que je la trouve ${ }^{47} . »$

Si Galiani a du génie, il offre, par son dialogisme, une manière qui satisfait à l'exigence épistémologique de présenter ses vues comme des possibilités à éprouver. À proprement parler, le dialogue exerce les forces du lecteur. Une allusion à Socrate dans l'Apologie atteste que Diderot prête une vertu éminemment pédagogique au dialogisme de

44 Voir Lester G. Crocker, «Le Neveu de Rameau, une expérience morale», dans Cahiers de l'Association internationale d'études françaises, 13, juin 1961, p. 133-156. Et «Jacques le fataliste, an «expérience morale»", dans Diderot Studies, t. 3, Syracuse University Press, 1961, p. 73-99.

45 Par exemple, Versini, t. 3, p. 141 : «De tout ce que j'ai lu jusqu'à présent, il m'a semblé que le Galiani a été souvent un raisonneur subtil et frivole, ce que l'abbé Morellet lui démontre bien, mais que celui-ci, ne s'appuyant sur aucun fait de détail, les méprisant même, est un raisonneur abstrait, utopique; que le Galiani connaît les hommes et les choses, que l'abbé Morellet ne connaît rien ; que le premier a de la gaieté, du style et du génie, que le second, dur, sec, ennuyeux, n'a rien de tout cela, et que sa Réfutation n'a pas rendu la question plus claire.» Je souligne.

46 Versini, t. 3, p. 130.

47 Versini, t. 1, par. 29, p. 28. 
Galiani, mais pédagogique au sens que Socrate donnait lui-même à sa maïeutique. Le Socrate de Diderot ${ }^{48} \mathrm{n}^{\prime}$ est pas le Socrate de La République de Platon: Diderot ne pense pas le dialogue comme la représentation ou la mise en scène d'une maïeutique, montrant un philosophe qui entraîne son interlocuteur vers une vérité dont il semble assuré qu'elle ait une préséance ontologique sur l'exercice du dialogue. Le dialogue, envisagé par Diderot, a une fonction performative, il fait office de véritable maïeutique pour son lecteur, dans la mesure où il le fait penser, ébranle, comme les renvois de l'Encyclopédie, les façons communes de penser. Diderot n'identifie justement pas le dialoguiste à Platon, mais à Socrate lui-même, ce qui sous-entend l'instauration d'un dialogue avec le lecteur. Plusieurs écrits de Diderot fonctionnent en effet sur ce registre, $s^{\prime}$ adressant directement au lecteur ${ }^{49}$, le plus souvent, sous forme d'interrogations, mais aussi par le truchement de «me direz-vous» et de «demandez-moi si». Diderot fait sans cesse exister un lecteur dynamique en face duquel il se situe, à qui il pose des questions, à qui il répond. En fait, le dialogisme de Diderot est multiforme et se caractérise surtout par le fait de cette présence constante du lecteur. Ainsi, à propos de Ceci n'est pas un conte, H.-R. Jauss ${ }^{50}$ fait remarquer que les deux histoires confrontées qui en forment le tissu et dont la force tient à leur unité respective «exigent du lecteur une réflexion sur les normes et critères de son jugement, ... mettent en question la morale sociale, ... la nature humaine, ... et la justice divine». Or, c'est évidemment là ce que Diderot entend lorsqu'il dit que le dialogue de Galiani sait nous faire penser. Du coup, la force d'un écrit même philosophique ne résiderait pas seulement dans son «énergie» interne, mais aussi dans le mouvement qu'il suscite chez celui qui le lit $t^{51}$.

48 Jean Seznec a montré comment Diderot s'est toute sa vie identifié à Socrate et ce, souvent même alors que Socrate était perçu comme un bouffon par ses proches. Voir «Le Socrate imaginaire», dans Essais sur Diderot et l'Antiquité, Oxford, Clarendon Press, 1957, p. 3-22.

49 Jacques le fataliste en est sans doute l'exemple le plus probant. Mais les Salons et de nombreux autres écrits jouent également avec le même genre d'effets.

50 Voir «Dialogique et dialectique (ou : Diderot lecteur de Socrate et Hegel lecteur de Diderot)», dans Revue de métaphysique et de morale, 1984, 2, p. 151.

51 Voir Colas Duflo, «Et pourquoi des dialogues en des temps de systèmes ?», dans Diderot Studies, t. 28, 2000, p. 95-109. 


\section{Conclusion}

Dans son livre sur le matérialisme de Diderot, Jean-Claude Bourdin écrit : «tout lecteur de Diderot vérifie que ses thèses matérialistes sont à ce point inséparables des ensembles discursifs variés et singuliers qui les prennent en charge, qu'il ne peut tenir pour un accident ou pour la seule expression d'un tempérament, le fait qu'il n'a réellement pas voulu construire et exposer dans ses traités une doctrine matérialiste ${ }^{52} . » \mathrm{Si}$ cela n'est pas accidentel, on l'a vu, c'est qu'il y va clairement d'un réquisit épistémologique. Celui de ne pas conférer aux idées plus de réalité qu'elles n'en peuvent avoir. Les idées, comme tous les êtres formant le «tout» de la nature, sont toujours, en un sens, locales et provisoires. Ce qui devrait signifier, Diderot en prend acte dans les Pensées sur l'interprétation de la nature, qu'il n'y a pas de philosophie.

Si les phénomènes ne sont pas enchaînés les uns aux autres, il n'y a point de philosophie. Les phénomènes seraient tous enchaînés, que l'état de chacun d'eux pourrait être sans permanence. Mais si l'état des êtres est dans une vicissitude perpétuelle ; si la nature est encore à l'ouvrage ; malgré la chaîne qui lie les phénomènes, il n'y a point de philosophie. Toute notre science naturelle devient aussi transitoire que les mots. Ce que nous prenons pour l'histoire de la nature n'est que l'histoire très incomplète d'un instant ${ }^{53}$.

Qu'il n'y ait pas de philosophie, cela signifie qu'il n'y a pas de savoir absolu, au sens d'un savoir total, d'une connaissance de toute chose ${ }^{54}$. La philosophie est à l'égal de cette nature, justement, que Diderot prend soin de définir : "le résultat général actuel, ou les résultats généraux successifs de la combinaison des éléments ${ }^{55}$.» Dès lors, tout génie qu'il soit, aucun être humain ne peut prétendre détenir le fin mot sur la nature. Ce qui fait, au fond, le véritable génie, ce n'est pas simplement le fait de voir loin dans la nature, mais sa capacité à nous faire voir au-delà de nous-mêmes, dans la région des possibles. Pour autant, la force du génie ne l'autorise pas à se dégager de l'exigence de vérité, seulement Diderot juge que le fait de faire vivre en nous le désir de se lancer à sa recherche

52 Jean-Claude Bourdin, Diderot. Le Matérialisme, Paris, PUF, 1998, p. 10.

53 Versini, t. 1, par. 58, p. 596-597.

54 Voir Sylvain Auroux, «Diderot encyclopédiste», dans Stanford French Review, automne 1984, p. 175-188. 
vaudra toujours plus, si tant est que l'on tienne compte, tout au long de cette recherche, des limites de l'esprit.

Partout, dans l'œuvre de Diderot, où surgit le génie, les catégories semblent bousculées. Nul autre que lui n'a la force de s'adresser à ce qu'il y a de plus universel dans la condition humaine, soit son désir de transcendance. Mais il importe qu'au cœur de cette adresse demeure un rappel de notre finitude. Si le génie peut conjecturer des mondes possibles, il doit toujours en manifester le caractère purement possible ; sans quoi sa génialité devient folie, tourne au dogme et, comme dirait Diderot, sa vivacité se perd et devient cette «morgue» qui s'associe toujours à la superstition. S'il y a une force capable d' «exercer» ou de «faire penser» dans l'œuvre philosophique de génie, c'est avant tout de manière performative, en tant qu'elle laisse voir sa propre incomplétude sur le fond d'une complétude possible : d'où l'importance des procédés discursifs dialogiques. Diderot, lorsqu'il écrit, engage toujours son lecteur à voir au-delà de son propre texte et exige de lui qu'il en éprouve pour lui-même la consistance.

MITIA RIOUX-BEAULNE

Université de Montréal 